\title{
Spectral-type dependent rotational braking and strong magnetic flux in three components of the late-M multiple system LHS 1070
}

\author{
A. Reiners ${ }^{1, \star}$, A. Seifahrt ${ }^{2,3}$, H. U. Käufl ${ }^{3}$, R. Siebenmorgen ${ }^{3}$, and A. Smette ${ }^{4}$ \\ 1 Universität Göttingen, Institut für Astrophysik, Friedrich-Hund-Platz 1, 37077 Göttingen, Germany \\ e-mail: Ansgar.Reiners@phys.uni-goettingen.de \\ 2 Universität Jena, Astrophysikalisches Institut und Instituts-Sternwarte, Schillergässchen 2, 07745 Jena, Germany \\ 3 European Southern Observatory, Karl-Schwarzschild-Str. 2, 85748 Garching, Germany \\ ${ }^{4}$ European Southern Observatory, Alonso de Córdova 3107, Casilla 19001, Santiago 19, Chile
}

Received 29 May 2007 / Accepted 14 June 2007

ABSTRACT

\begin{abstract}
We show individual high-resolution spectra of components $\mathrm{A}, \mathrm{B}$, and $\mathrm{C}$ of the nearby late-M type multiple system LHS 1070 . Component $\mathrm{A}$ is a mid-M star, and $\mathrm{B}$ and $\mathrm{C}$ are known to have masses at the threshold to brown dwarfs. From our spectra we measure rotation velocities and the mean magnetic field for all three components individually. We find magnetic flux on the order of several kilo-Gauss in all components. The rotation velocities of the two late-M objects B and C are $\operatorname{similar}\left(v \sin i=16 \mathrm{~km} \mathrm{~s}^{-1}\right)$, the earlier A component is spinning only at about half that rate. This suggest that net rotational braking at late-M spectral type is weakening, and that the lack of slowly rotating late-M and $\mathrm{L}$ dwarfs is real. Furthermore, we found that magnetic flux in the B component is about twice as strong as in component $\mathrm{C}$ at a similar rotation rate. This indicates that rotational braking is not proportional to magnetic field strength in fully convective objects and that a different field topology is the reason for the weak braking in low-mass objects.
\end{abstract}

Key words. stars: low-mass, brown dwarfs - stars: magnetic fields - stars: rotation - stars: individual: LHS 1070

\section{Introduction}

The rotation of Sun-like stars is braked following an empirically determined braking law with $\boldsymbol{v} \propto t^{-0.5}$ (Skumanich 1972; Barnes 2007). At several Gyr they have lost most of their angular momentum and become slow rotators like the Sun. Fully convective stars, however, are apparently not braked that much, and field dwarfs of spectral type late-M or L rotate more rapidly than their higher mass siblings (Mohanty \& Basri 2003).

Stars are believed to lose angular momentum due to a magnetic stellar wind after the first phase of star formation. Angular momentum loss critically depends on the star's magnetic field and its geometry (Mestel 1984; Kawaler 1988; Chaboyer et al. 1995; Sills et al. 2000). The mechanism of magnetic field generation is of crucial importance since it determines magnetic field strength, hence braking, and its reaction to angular velocity changes. Since the mechanism of magnetic field generation is believed to change at the threshold to fully convective stars, a change in the braking law appears can be expected as well.

The lack of slowly rotating objects with a spectral type later than mid-M could be the consequence of a change in the net braking. These objects, however, are so faint that it is difficult to probe a sample that is unbiased to luminosity effects, i.e. a luminosity-limited sample will always contain more bright (hence young and less braked) low-mass objects than faint ones.

The close, hierarchical system LHS 1070 (GJ 2005) is an ideal probe for rotational evolution of late-M objects. Its individual components can be spatially resolved; the A component is a mid-M star (M5.5) while the two fainter components B and $\mathrm{C}$ are cooler (around M9). Measuring the astrometric orbit of B

\footnotetext{
* Emmy Noether Fellow.
}

and C, Leinert et al. (2000) determined masses at the limit of brown dwarfs.

In this paper, we measure the rotation velocities and magnetic flux of LHS $1070 \mathrm{~A}, \mathrm{~B}$, and C individually. Because all components are probably coeval, this gives direct insight into spectral-type dependent braking and magnetic-field generation.

\section{Data}

In 2006, the three components LHS $1070 \mathrm{~A}, \mathrm{~B}$, and C were almost exactly lined up on the sky, a most suitable configuration for simultaneous long-slit spectroscopy. We observed the triple on the night of October 9, 2006 during a science verification run (Prog.ID 60.A-9078) of the newly commissioned AO-fed highresolution NIR spectrograph CRIRES (Käufl et al. 2006) at Antu (UT1) of the VLT on Cerro Paranal.

Separations of LHS $1070 \mathrm{AB}$ and AC are about 1.35" and $1.75^{\prime \prime}$, respectively. The spectra are spatially well-resolved with only minimal overlap of the $\mathrm{B}$ and $\mathrm{C}$ components. Observations were carried out in order 57/0/i with on-target $\mathrm{AO}$, and the total exposure time was $480 \mathrm{~s}$. We chose a slitwidth of $0.4^{\prime \prime}$, i.e. a nominal resolving power of 50000 . Data reduction followed the standard procedure of dark subtraction and flatfielding. After proper background subtraction to remove the $\mathrm{OH}$ airglow emission, the spectra were extracted using an adaption of the optimal extraction algorithm of Robertson (1986). The chosen spectral window is clear of telluric lines at a $2 \%$ level. Thus we do not need to use a standard star for correction. Instead we determined the instrument response from the flatfield spectra and removed the remaining tilt by minimal rectification of the pseudocontinuum. 


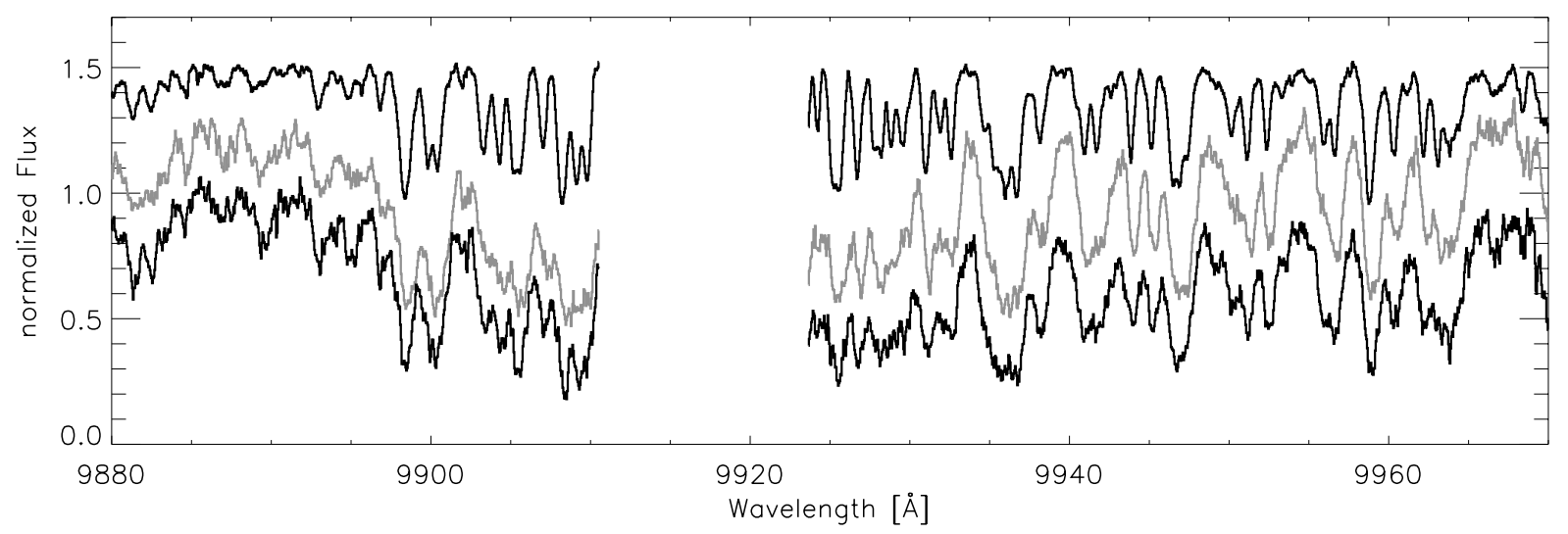

Fig. 1. A part of our CRIRES data in the three components LHS 1070 A, B, and C (from top to bottom), smoothed by a 3 pixel box-car.

Wavelength calibration was performed using a ThAr spectrum in comparison to an updated line list (Kerber, priv. comm.). Flux contamination of the $\mathrm{B}$ and $\mathrm{C}$ components was found to be much smaller than the intrinsic $\mathrm{S} / \mathrm{N}$ ratio. Thus we do not see any reason to doubt the spectral purity of any of the components in LHS 1070 for the subsequent analysis. A part of our data is shown in Fig. 1, where at $9920 \AA$ the spectrum falls on a gap between two of the four chips.

\section{Analysis and results}

Our observations were carried out in the Wing-Ford band of molecular FeH (starting at $9900 \AA$ ), which shows sharp absorption features in mid- to late-M dwarfs. Some of the lines are very magnetically sensitive, while some are not (Reiners \& Basri 2006b). This makes the region ideal for analyzing rotational broadening and measuring magnetic fields in ultra-cool objects. Our analysis follows the strategy used by Reiners \& Basri (2007) and Reiners et al. (2007).

The method uses spectra of two template M-stars, one without any signs of Zeeman broadening and the other showing the strong effects of Zeeman broadening. First, the template spectra are scaled according to an optical depth scaling law to fit the target's absorption strength. Then, the two template spectra are artificially broadened to match the target's rotation velocity. Finally, we search for the linear combination of the two template spectra that simultaneously fits the magnetically sensitive and the magnetically insensitive lines. From the interpolation parameter, we determine $B f$, the product of the mean magnetic field and the filling factor, assuming a linear relation between Zeeman broadening and $B f$ (see Reiners \& Basri 2006b, 2007).

As template spectra, we use data of GJ 1002 (M5.5) and G1 873 (M3.5) taken with HIRES at Keck observatory ${ }^{1}$, both stars have $v \sin i \leq 3 \mathrm{~km} \mathrm{~s}^{-1}$. Because the template spectra were taken at lower spectral resolving power $(R=31000)$, we apply a Gaussian broadening function to our data in order to match the resolution of the templates. This is a crucial step since it sets the zero point for the calibration of rotational broadening. In order to check our results, we carry out the analysis with a different set of templates observed at higher resolution. We used a spectrum of GJ 1227 (M4.5, $v \sin i \leq 3 \mathrm{~km} \mathrm{~s}^{-1}$ ) for the field-free template and a sunspot spectrum for the spectrum in the presence of a magnetic field ${ }^{2}$. The spectrum of GJ 1227 was also taken with HIRES at Keck but at a higher resolving power $(R \sim 80000)$.

\footnotetext{
1 We thank G. Basri for providing the template spectra.

2 ftp://ftp.noao.edu
}

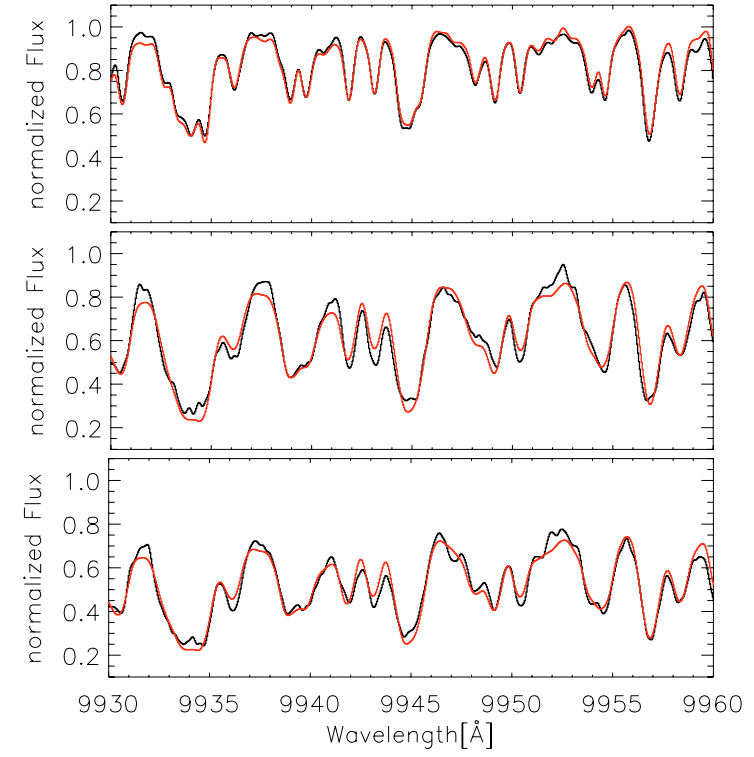

Fig. 2. Data (black) and fit (red) in the entire region used for the fit in the three components LHS 1070, A, B, and C (from top to bottom). The data was degenerated in resolution to match the resolution of the template spectra $(R=31000)$.

The template spectra are degenerated in order to match the resolution of our CRIRES data. We find that both strategies provide highly consistent results for the values of $v \sin i$ for all three components of LHS 1070; i.e. differences are within $1 \mathrm{~km} \mathrm{~s}^{-1}$. For the following, we rely on the low-resolution set of templates because they provide a much more consistent data set, and their resolution is entirely sufficient for our purposes.

\subsection{Rotation}

We show our best fits over the $30 \AA$-wide region used for fitting as red lines in all three components of LHS 1070 in Fig. 2. Smoothing our spectra to match the lower resolution of the template spectra strongly reduced the effective noise level. The $\mathrm{S} / \mathrm{N}$ per resolution element are now on the order of 70 for LHS 1070 A, 35 and 30 for LHS 1070 B and C, respectively. Differences in projected rotation velocity can be distinguished by eye for example at $9939 \AA$ where two neighbored lines are just separated in the A-component but already blended in components B and C. The two latter objects do not show any obvious difference in the width of individual lines. 


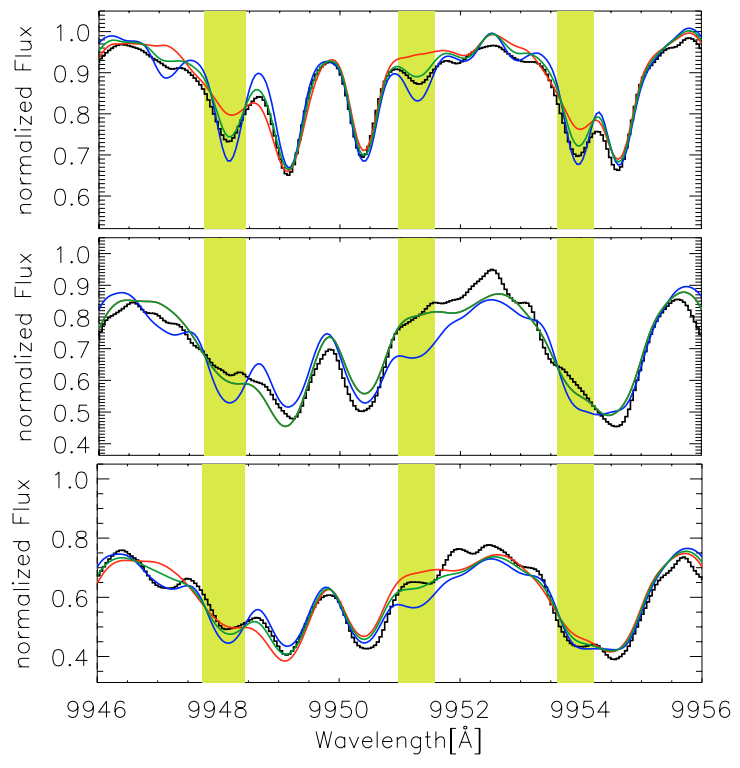

Fig. 3. Portion of the spectral range that shows the effect of magnetic flux. Green bars mark the magnetically most sensitive lines. Top to bottom: LHS $1070 \mathrm{~A}, \mathrm{~B}$, and C. Black lines are the target spectra, red lines mark spectra affected by strong magnetic flux (Gl 873), and blue lines spectra without magnetic flux (GJ 1002). Green lines are our best fits. Note that in the B component the red line is covered by the green one.

We derive projected rotation velocities of $v \sin i=8,16$, and $16 \mathrm{~km} \mathrm{~s}^{-1}$ in LHS $1070 \mathrm{~A}, \mathrm{~B}$, and C, respectively. The overall fit quality is rather good particularly in the A-component of the system. In the two late-M components $\mathrm{B}$ and $\mathrm{C}$, the fit has some weaknesses that we attribute to the much stronger absorption that may not be perfectly captured by the optical depth scaling. However, for our analysis of $v \sin i$ the quality is entirely sufficient. A formal $\chi^{2}$ analysis shows that the uncertainty in $v \sin i$ is on the order of $1 \mathrm{~km} \mathrm{~s}^{-1}$ for the three objects. The absolute uncertainty is somewhat higher since the zero-point depends on the resolution we adopt for the template spectra and our target spectra. We estimate that absolute uncertainties are on the order of $3 \mathrm{~km} \mathrm{~s}^{-1}$, but that does not affect the comparison of rotation velocities between the three components of LHS 1070. Basri \& Marcy (1995) have measured $v \sin i$ in a spatially unresolved spectrum of LHS 1070. They find $v \sin i=8 \pm 2 \mathrm{~km} \mathrm{~s}^{-1}$ in excellent agreement with our result for LHS $1070 \mathrm{~A}$, which dominates the spatially unresolved spectrum.

\subsection{Magnetic flux}

Magnetic flux was determined by searching for a linear interpolation between a field free template spectrum and a template spectrum affected by magnetic Zeeman broadening. For the latter, we use a spectrum of Gl 873 and we adopt a magnetic flux of $B f=3.9 \mathrm{kG}$ (see Reiners \& Basri 2007). This implies that our results cannot exceed values of $\sim 4 \mathrm{kG}$.

At every wavelength, the interpolation lies between the two template spectra. In magnetically insensitive regions, the difference is very small while at wavelengths that are affected by Zeeman broadening, a difference proportional to the magnetic flux occurs. We show a representative region of our spectra at the wavelength range 9946-9956 ̊ in Fig. 3. Here, we highlight three absorption lines that are particularly sensitive to Zeeman broadening. We plot the target spectra in black and overplot the (rotationally broadened) template spectra of GJ 1002 (no
Table 1. Parameter of the three components LHS 1070 A, B, and C.

\begin{tabular}{lccccc}
\hline \hline Component & SpType $^{a}$ & $V$ & $J$ & $\begin{array}{c}v \sin i \\
{\left[\mathrm{~km} \mathrm{~s}^{-1}\right]}\end{array}$ & $\begin{array}{c}B f \\
{[\mathrm{kG}]}\end{array}$ \\
\hline LHS 1070 A & M 5.5 & 15.42 & 9.25 & 8 & 2.0 \\
LHS 1070 B & M 8.5 & & & 16 & 4.0 \\
LHS 1070 C & M 9.0 & & & 16 & 2.0 \\
\hline
\end{tabular}

${ }^{a}$ From Leinert et al. (2000).

magnetic flux) in blue, and the one of Gl 873 (strong flux) in red. The interpolation that best fits the data is overplotted in green.

A very accurate fit is achieved in the spectrum of LHS 1070 A, from which we derive a magnetic flux of $2.0 \mathrm{kG}$. The fit quality in the spectrum of LHS $1070 \mathrm{~B}$ is not as accurate. However, it appears that the two sensitive absorption lines at $9948 \AA$ and $9954 \AA$ are strongly smeared out, possibly even more than in the template spectrum of Gl 873 . This indicates very strong magnetic flux, and we adopt a value of $B f \sim 4 \mathrm{kG}$ for component $\mathrm{B}$ (note that the red line is covered by the green fit in this panel). The presence of strong magnetic flux can also be seen by comparing the spectrum of LHS $1070 \mathrm{~B}$ to the one of LHS $1070 \mathrm{C}$ in the third panel. Here, the fit quality is much higher again, we deduce a magnetic flux of $2 \mathrm{kG}$ as in the A-component. Comparing this spectrum to the one of LHS 1070 B (particularly the lines at $9948 \AA$ and $9954 \AA$ ), it appears that the magnetically sensitive absorption lines are much weaker in LHS 1070 B than they are in LHS 1070 C.

As discussed in Reiners \& Basri (2006b, 2007), our lack of knowledge about the Zeeman splitting patterns of the $\mathrm{FeH}$ molecule causes the main uncertainty in the determination of magnetic flux using this molecule as a tracer. On an absolute scale, we estimate our uncertainties in magnetic flux on the order of a kilo-Gauss. However, the main result of our magnetic flux analysis is already seen in a rough comparison of the three spectra: All three components show a substantial effect of magnetic flux on the spectral lines, and the magnetic flux in LHS $1070 \mathrm{~B}$ is stronger than the flux in LHS 1070 A and LHS 1070 C.

\section{Discussion and conclusions}

We have isolated high-resolution spectra of the three components of the multiple M-object system LHS 1070. From each spectrum, we determined the projected rotation velocity $v \sin$ and the mean magnetic field over the entire star. The results of our analysis are summarized in Table 1. We find a projected rotational velocity of $v \sin i=8 \mathrm{~km} \mathrm{~s}^{-1}$ in the hottest component, LHS $1070 \mathrm{~A}$, while rotation in LHS $1070 \mathrm{~B}$ and C is about twice as rapid. Under the reasonable assumption that the entire system LHS 1070 was formed at the same time, we are facing three different objects that have evolved from the same initial conditions. Disc orientations in pre-main sequence stars (Monin et al. 2006) and orbit orientations in multiple systems (Sterzik \& Tokovinin 2002) are partially correlated, and Bate et al. (2000) find that strong misalignments are unlikely in binaries with separations $\leq 100 \mathrm{AU}$. Thus, we may assume that the inclination angles $i$ of LHS $1070 \mathrm{~A}, \mathrm{~B}$, and C are comparable (see also Leinert et al. 2001). As long as they suffer the same rotational braking, we would expect comparable rotation velocities in all components. We observe comparable rotation rates in the components $\mathrm{B}$, and $\mathrm{C}$, which are of very similar spectral type. However, the rotation velocity in LHS $1070 \mathrm{~A}$ is about a factor of two lower. This can probably be attributed to a difference in the rotational braking during their evolution. 


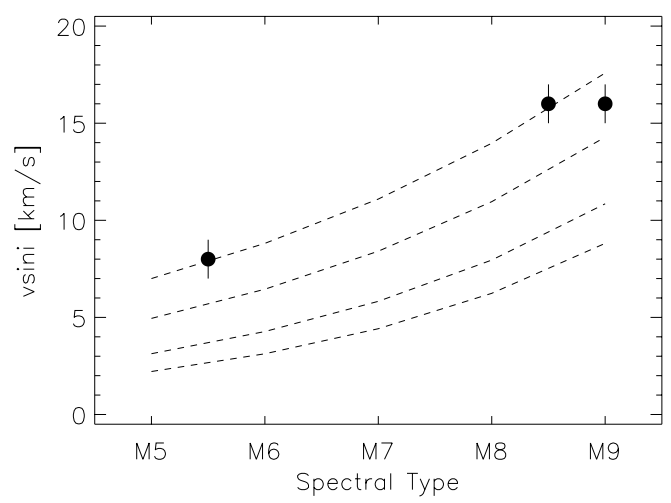

Fig. 4. Projected rotation velocities of LHS 1070 A, B, and C. We overplot rotation velocities according to a modified Skumanich braking law at 1,2,5, and $10 \mathrm{Gyr}$ (top to bottom).

Mohanty \& Basri (2003) show that the fraction of slow rotators $\left(v \sin i<3 \mathrm{~km} \mathrm{~s}^{-1}\right.$ ) later than spectral type M6 in the field is much smaller than among early-M dwarfs, which generally rotate very slowly (Delfosse et al. 1998). However, the sample of Mohanty \& Basri (2003) could be biased to bright (hence young) objects that are still rotating more rapidly than their older (hence fainter) siblings. Reiners \& Basri (2006a) find slow rotation in a mid/late-M subdwarf, and very rapid rotation ( $v \sin i=65 \mathrm{~km} \mathrm{~s}^{-1}$ ) in a late-L subdwarf. Since late-type subdwarfs are probably the oldest relics of the early galaxy having spun down for several Gyr, this is another argument for less efficient rotational braking for a later spectral type.

In LHS 1070, we see three objects of different spectral type that are probably of same age. We plot the projected rotation velocities of LHS $1070 \mathrm{~A}, \mathrm{~B}$, and C in Fig. 4. As stated above, the two later objects show higher rotation rates than the earlier A-component. One explanation may be a difference in the initial conditions, but together with the results by Mohanty \& Basri (2003), this would suggest that later objects generally start off by rotating more rapidly, which is unlikely. On the other hand, the rotation-activity connection seems to vanish around late-M spectral type. This is probably connected to a modified wind law and consequently to different rotational braking.

Detailed simulations of rotational braking were performed by Chaboyer et al. (1995) and Sills et al. (2000). They give a prescription for a wind-braking law in stars at a given angular velocity, and they show that the braking law in fully convective stars requires different treatments (to fit observations in the Hyades they had to tune their value of $\omega_{\text {crit }}$; Sills et al. 2000). In this work, we have not intended to give a full description of the braking law in fully convective objects. Instead, we assume that the net braking law in LHS $1070 \mathrm{~A}$ is still Skumanich-type $\left(v \propto t^{-0.5}\right.$, Skumanich 1972) and that the exponent of the braking law is gradually decreasing towards lower temperatures.

For this purpose, we calculate the rotational evolution starting at an age of $10 \mathrm{Myr}$ and an initial rotation velocity of $70 \mathrm{~km} \mathrm{~s}^{-1}$. For our spectral-type dependent braking law, we chose $v \propto t^{-\alpha}$ with gradually decreasing $\alpha=0.5-0.3$ in the spectral range M5-M9. Our results are overplotted in Fig. 4. Four lines for ages of 1, 2, 5, and $10 \mathrm{Gyr}$ are shown (top to bottom). The assumed spectral-type dependent scaling law can reproduce the difference in rotation velocities as measured in LHS 1070 and, at the same time, generate the rise in minimum rotational velocities consistent with measurements in late-M stars (Mohanty \& Basri 2003). We thus conclude that braking in late-M stars is spectral-type dependent, and that the lack of slowly rotating late-M dwarfs is not a selection effect due to a bias towards young rapid rotators.

The age estimated from our approach is on the order of 1 Gyr. Basri \& Marcy (1995) found a high-velocity component perpendicular to the galactic plane that suggests higher age. We think that this point deserves further attention. Although age estimates from space velocity are rather uncertain, this may hint at an absolute scale of the braking law different to the one used here.

All three components of LHS 1070 show strong magnetic flux, suggesting that in fully convective objects, the field strength does not strongly depend on rotation. Leinert et al. (2000) found signs of magnetic activity in components A and B, but not in C. This is consistent with our results in the sense that the magnetic flux in LHS $1070 \mathrm{~B}$ is much stronger than the one in LHS 1070 C. Nevertheless, one expects that the C component also exhibits $\mathrm{H} \alpha$ emission, which may still be below the detection limit of Leinert et al. (2000).

Interestingly, the difference in magnetic flux between LHS $1070 \mathrm{~B}$ and C apparently does not influence rotational braking. It is generally believed that angular momentum loss depends on magnetic field strength with an exponent given by the field geometry (Mestel 1984). This coupling, however, depends on the field generation mechanism (Kawaler 1988), and angular momentum loss becomes saturated at a certain level (Chaboyer et al. 1995). According to Chabrier \& Küker (2006), large-scale magnetic fields of equipartition strength (a few $\mathrm{kG}$ ) can be generated by an $\alpha^{2}$ dynamo. These authors predict that the field topology differs from an organized dipole field, which could explain our result that rotational braking does not grow with magnetic field strength. It would imply that field geometry is the reason for lower rotational braking in low-mass objects.

Acknowledgements. We would like to thank the CRIRES science verification team for their work on CRIRES and for the execution of the observations, and the referee, Subu Mohanty, for a very constructive report. A.R. acknowledges financial support through an Emmy Noether Fellowship from the Deutsche Forschungsgemeinschaft under DFG RE 1664/4-1.

\section{References}

Barnes, S. A. 2007, ApJ, in press [arXiv: astro-ph:0704. 3068] Basri, G., \& Marcy, G. W. 1995, AJ, 109, 762

Bate, M. R., Bonnell, I. A., Clarke, C. J., et al. 2000, MNRAS, 317, 773 Chaboyer, B., Demarque, P., \& Pinsonneault, M. H. 1995, ApJ, 441, 875 Chabrier, G., \& Küker, M. 2006, A\&A, 446, 1027

Delfosse, X., Forveille, T., Perrier, C., \& Mayor, M. 1998, A\&A, 331, 581 Käufl, H. U., et al. 2006, The Messenger, 126, 32

Leinert, C., Allard, F., Richichi, A., \& Hauschildt, P. H. 2000, A\&A, 353, 691 Leinert, C., Jahreiß, H., Woitas, J., et al. 2001, A\&A, 367, 183

Kawaler, S. D. 1988, ApJ, 333, 236

Mestel 1984, in Third Cambridge Workshop on Cool Stars, Stellar Systems, and the Sun, ed. S. L. Baliunas, \& L. Hartmann (New York: Springer), 49 Mohanty, S., \& Basri, G. 2003, ApJ, 583, 451

Monin, J.-L., Mènard, F., \& Peretto, N. 2006, A\&A, 446, 201

Reiners, A., \& Basri, G. 2006a, AJ, 131, 1806

Reiners, A., \& Basri, G. 2006b, ApJ, 644, 497

Reiners, A., \& Basri, G. 2007, ApJ, 656, 1121

Reiners, A., Schmitt, J. H. M. M., \& Liefke, C. 2007, A\&A, 466, L13

Robertson, J. G. 1986, PASP, 98, 1220

Sills, A., Pinsonneault, M. H., \& Terndrup, D. M. 2000, ApJ, 534, 335

Skumanich, A. 1972, ApJ, 171, 565

Sterzik, M. F., \& Tokovinin, A. A. 2002, A\&A, 384, 1030 